\title{
AGILE-ACCORD: A Randomized, Multicentre, Seamless, Adaptive Phase I/II Platform Study to Determine the Optimal Dose, Safety and Efficacy of Multiple Candidate Agents for the Treatment of COVID-19: A structured summary of a study protocol for a randomised platform trial
}

Gareth Griffiths $^{1 *}$, Richard Fitzgerald ${ }^{2}$, Thomas Jaki ${ }^{3}$, Andrea Corkhill ${ }^{4}$, Ellice Marwood ${ }^{4}$, Helen Reynolds ${ }^{5}$, Louise Stanton ${ }^{4}$, Sean Ewings ${ }^{4}$, Susannah Condie ${ }^{4}$, Emma Wrixon ${ }^{4}$, Andrea Norton ${ }^{6}$, Mike Radford ${ }^{4}$, Sara Yeats ${ }^{4}$, Jane Robertson ${ }^{4}$, Rachel Darby-Dowman ${ }^{7}$, Lauren Walker ${ }^{5}$, Saye Khoo ${ }^{5}$ and on behalf of the UK NIHR community

\begin{abstract}
Objectives: Phase I - To determine the optimal dose of each candidate (or combination of candidates) entered into the platform.

Phase II - To determine the efficacy and safety of each candidate entered into the platform, compared to the current Standard of Care (SoC), and recommend whether it should be evaluated further in a later phase II \& III platforms.

Trial design: AGILE-ACCORD is a Bayesian multicentre, multi-arm, multi-dose, multi-stage open-label, adaptive, seamless phase I/II randomised platform trial to determine the optimal dose, activity and safety of multiple candidate agents for the treatment of COVID-19. Designed as a master protocol with each candidate being evaluated within its own sub-protocol (Candidate Specific Trial (CST) protocol), randomising between candidate and SoC with 2:1 allocation in favour of the candidate (N.B the first candidate has gone through regulatory approval and is expected to open to recruitment early summer 2020). Each dose will be assessed for safety sequentially in cohorts of 6 patients. Once a phase II dose has been identified we will assess efficacy by seamlessly expanding into a larger cohort.

(Continued on next page)
\end{abstract}

\footnotetext{
* Correspondence: G.O.Griffiths@soton.ac.uk

${ }^{1}$ University of Southampton, Southampton, Hampshire, UK

Full list of author information is available at the end of the article
}

C C The Author(s). 2020 Open Access This article is licensed under a Creative Commons Attribution 4.0 International License, which permits use, sharing, adaptation, distribution and reproduction in any medium or format, as long as you give appropriate credit to the original author(s) and the source, provide a link to the Creative Commons licence, and indicate if changes were made. The images or other third party material in this article are included in the article's Creative Commons. licence, unless indicated otherwise in a credit line to the material. If material is not included in the article's Creative Commons licence and your intended use is not permitted by statutory regulation or exceeds the permitted use, you will need to obtain permission directly from the copyright holder. To view a copy of this licence, visit http://creativecommons.org/licenses/by/4.0/. The Creative Commons Public Domain Dedication waiver (http://creativecommons.org/publicdomain/zero/1.0/) applies to the data made available in this article, unless otherwise stated in a credit line to the data. 
(Continued from previous page)

Participants: Patient populations can vary between CSTs, but the main eligibility criteria include adult patients ( $\geq 18$ years) who have laboratory-confirmed infection with severe acute respiratory syndrome coronavirus 2 (SARS-CoV-2). We will include both severe and mild-moderate patients defined as follows: Group A (severe disease) - patients with WHO Working Group on the Clinical Characteristics of COVID-19 infection 9-point ordinal scale of Grades 4 (hospitalised, oxygen by mask or nasal prongs), 5 (hospitalised, non-invasive ventilation or high flow oxygen), 6 (hospitalised, intubation and mechanical ventilation) or 7 (hospitalised, ventilation and additional organ support); Group B (mild-moderate disease) - ambulant or hospitalised patients with peripheral capillary oxygen saturation $\left(\mathrm{SpO}_{2}\right)>94 \%$ RA. If any CSTs are included in the community setting, the CST protocol will clarify whether patients with suspected SARS-CoV-2 infection are also eligible.

Participants will be recruited from England, North Ireland, Wales and Scotland.

Intervention and comparator: Comparator is the current standard of care (SoC), in some CSTs plus placebo. Candidates that prevent uncontrolled cytokine release, prevention of viral replication, and other anti-viral treatment strategies are at various stages of development for inclusion into AGILE-ACCORD. Other CSTs will be added over time. There is not a set limit on the number of CSTs we can include within the AGILE-ACCORD Master protocol and we will upload each CST into this publication as each opens to recruitment.

Main outcomes: Phase I: Dose limiting toxicities using Common Terminology Criteria for Adverse Events v5 Grade $\geq 3$ adverse events.

Phase II: Agreed on a CST basis depending on mechanism of action of the candidate and patient population. But may include; time to clinical improvement of at least 2 points on the WHO 9-point category ordinal scale [measured up to 29 days from randomisation], progression of disease (oxygen saturation $\left(\mathrm{SaO}_{2}\right)<92 \%$ ) or hospitalization or death, or change in time-weighted viral load [measured up to 29 days from randomisation].

Randomisation: Varies with CST, but default is 2:1 allocation in favour of the candidate to maximise early safety data.

Blinding (masking): For the safety phase open-label although for some CSTs may include placebo or SoC for the efficacy phase.

Numbers to be randomised (sample size): Varies between CSTs. However simulations have shown that around 16 participants are necessary to determine futility or promise of a candidate at a given dose (in efficacy evaluation alone) and between 32 and 40 participants are required across the dose-finding and efficacy evaluation when capping the maximum number of participants contributing to the evaluation of a treatment at 40.

Trial Status: Master protocol version number v5 07 May 2020, trial is in setup with full regulatory approval and utilises several digital technology solutions, including Medidata's Rave EDC [electronic data capture], RTSM for randomisation and patient eConsent on iPads via Rave Patient Cloud. The recruitment dates will vary between CSTs but at the time of writing no CSTs are yet open for recruitment.

Trial registration: EudraCT 2020-001860-27 14 th $^{\text {March }} 2020$

Full protocol: The full protocol is attached as an additional file, accessible from the Trials website (Additional file 1). In the interest in expediting dissemination of this material, the familiar formatting has been eliminated; this Letter serves as a summary of the key elements of the full protocol.

Keywords: COVID-19, Randomised controlled trial, Platform study, Master protocol, Phase I/II, Bayesian

\section{Supplementary information}

Supplementary information accompanies this paper at https://doi.org/10. 1186/s13063-020-04473-1.

Additional file 1. Full protocol.

\section{Acknowledgements}

We acknowledge Cancer Research UK (CRUK) who, during the COVID-19 crisis, allowed its staff at Southampton CTU to work with the National Institute for Health Research (NIHR) community (e.g. Southampton CTU, Liverpool CRF \& Southampton Biomedical Research (entre) to develop this phase I/II trial platform. We also acknowledge the University Hospital Southampton
NHS Foundation Trust, Liverpool University Hospitals NHS Foundation Trust, University of Southampton and the University of Liverpool who supported and collaborated in the development of AGILE-ACCORD.

\section{Authors' contributions}

GG, SK and RF came up with the concept of the development of AGILEACCORD and oversaw the development of the master protocol and this submission. TJ was responsible for the development of the statistical design and SE/LS the statistical aspects of running and analysing the trial. EM, AC, $H R$, LW were responsible for developing the trial management and delivery aspects of the trial. SC, EW, AN, MR and RDD were responsible for the development of data collection and management aspects of the trial. SY was responsible for quality assurance and pharmacovigilance aspects of the study and JR operational aspects. All were involved in the development of the 
master protocol and this submission. The author(s) read and approved the final manuscript.

\section{Authors' information}

GG (Director of the NIHR/CRUK Southampton Clinical Trials Unit), RF (Director of the NIHR Clinical Research Facility (CRF) in Liverpool) and SK (Chief Investigator of AGILE-ACCORD) have developed this master protocol for, and on behalf, the UK NIHR and CRF community and COVID-19 patients. Although we intend to run AGILE-ACCORD in the UK we invite researchers around the world (especially low and middle income countries) to use our master protocol as a template to help conduct their own COVID-19 research in their own countries.

\section{Funding}

The development of the AGILE-ACCORD Master Protocol, electronic data capture system, randomisation and econsent was funded using Cancer Research UK core funding and NIHR CTU support funding at the Southampton Clinical Trials Unit. NIHR funding at the Royal Liverpool and Broadgreen Clinical Research Facility and staff at the University of Liverpool and Lancaster also supported the development of the master protocol and its associated documentation (eg participant information sheets, case report forms). Funding for each Candidate Specific Trial protocol is expected to be a mixture of NIHR, UKRI and investigator initiated research grants from pharmaceutical companies supplying the candidate.

\section{Availability of data and materials}

When the database from each Candidate Specific Trial Protocol is locked, analysed and published we will make the data available to the academic community via www.clinicalstudydatarequest.com

\section{Ethics approval and consent to participate}

West Midlands - Edgbaston Research Ethics Committee (REC reference: 20/WM/0136), 12/05/2020, https://www.hra.nhs.uk/planning-and-improvingresearch/application-summaries/research-summaries/agile-accordaccelerating-covid-19-drug-development-covid-19-uph/,

I certify that this trial has received appropriate ethical approval as described above.

We will obtain consent from all participants entering into AGILE_ACCORD. If the patient lacks capacity to give consent due to the severity of their medical condition (e.g. patients with WHO clinical severity scores of 6 (hospitalised, intubation and mechanical ventilation) and 7 (ventilation and additional organ support - pressors, renal replacement therapy (RRT), extracorporeal membrane oxygenation (ECMO)), then in the first instance, consent may be obtained from the patient's personal legal representative.

\section{Consent for publication}

Not applicable.

\section{Competing interests}

The authors declare that they have no competing interests.

\section{Author details}

${ }^{1}$ University of Southampton, Southampton, Hampshire, UK. ${ }^{2}$ Liverpool University Hospitals NHS Foundation Trust, Liverpool, UK. ${ }^{3}$ Lancaster University and University of Cambridge, Lancaster, Lancashire, UK. ${ }^{4}$ Southampton CTU, University of Southampton, Southampton, Hampshire, UK. ${ }^{5}$ University of Liverpool, Liverpool, UK. ${ }^{6}$ Medidata Solutions, London, UK.

${ }^{7}$ Cancer Research UK Centre for Drug Development, London, UK.

Received: 27 May 2020 Accepted: 30 May 2020

Published online: 19 June 2020

\section{Publisher's Note}

Springer Nature remains neutral with regard to jurisdictional claims in published maps and institutional affiliations. 